\title{
Research on Internet of system based on communication network
}

\author{
Jinhai Zhang
}

Marine college of Shandong jiaotong university, Weihai, Shandong, China

Keywords: The Internet of things, communications networks, congestion control, VPDN

\begin{abstract}
In various fields of application of Internet of things endlessly, it development paints a bright future. However, the Internet application of common standards are not unified, compatibility between different Internet systems and scalable, these shortcomings and irregularities are restricted the application of Internet of popularization and the degree of interconnection. Internet terminals for different applications in different fields can be unified access to the communication backbone, thus achieving uniform standards of transmission network. In General, hosted by the communication network for the Internet of things to achieve and to provide material and material, objects and people, people and multiple modes of communication between people.
\end{abstract}

\section{Introduction}

IOT has important practical significance and commercial value, has been focusing on hot issues in the world, one of the study on China's Internet of things to a strategic height. Internet of things Internet is the second after another wave of informatization development in the fields of information industry, is capable of intelligent sensor terminal connection of all walks, integration of information and communication technologies, providing individual and industry-oriented applications and services, has a broad prospect.

China's Internet is still in its initial stage of research and development, technology is not yet mature, the industrial chain is not complete, but due to the rapid development of 3G technology and mature, traditional voice and data services markets increasingly saturated, in the communication industry chain service providers, equipment manufacturers, operators are focusing on the Internet. For operators, using the 3G network to carry Internet business has both technical and market advantages, and to expand market share, access to development opportunities.

Current of real networking application system, General are is a isolated closed of structure, no a common of technology standard, mutual Zhijian cannot formed unified of Internet, various sensor or network facilities also cannot get effective of using, so in real networking of design and application Shang compared single and low effect, caused more of repeat resources of investment, such also on cannot full of using public based equipment for social provides service. In traditional thought, always the underlying communication infrastructure or communications services with separate treatment and other public infrastructure.

Through analysis communications network on real networking business of hosted, combined information technology and the field of physical based facilities, proposed a based on VPDN of real networking standard achieved system, the standard can effective of solution due to standard not unified, and access platform not unified by brings of all problem, will real networking can and fusion communications technology and other the field of technology fusion up, this advanced of thinking and standard has is important of meaning.

\section{The existing communications network's Internet business}

Internet of things is any need of a Terminal, via radio frequency identification or sensors connected to the Internet and a network for data sharing and Exchange. Internet remote monitoring and management, tracking, field data collection and survey, and to the Internet terminal management and control purposes. Hosted on 3G or on the Internet, Internet business, complete objects and people, people and people, real-time information exchange between substance and, make the person or thing on the "demand" access to information, achieve broad unity of intelligent 
network information realize world of information "great harmony".

Essentially, the Internet of things is based on communication network to host a new carrier, Omni-directional, point of Pan-wide communication and capacity. In future communications from Internet business traffic, data traffic for a large proportion of the total. Therefore, hosts of the communication network or the Internet traffic and hosts have a significant impact on the way.

Internet business types of complexity, breadth and diversity on network capacity and a higher quality of service requirements. IOT hosted network requires additional carrier-grade features for network quality of service, security, controllable tube presented very high demands. In order to meet the Internet business hosting, needs to further improve the access network, metropolitan area network, backbone carrier-class requirements, including end-to-end quality of service, network's self-healing capacity, business protection, network security, and so on. Internet architecture can be divided into three layers, from top to bottom for the sensing layer, the transport layer and application layer.

From the current Internet applications in various fields, basically can be classified into four types, namely, emergency monitoring, data acquisition and transmission category, video surveillance, intelligent identification.

\section{Internet system scheme based on VPDN}

Common Internet system consists of three parts. First part is the front end capture devices, sensor networks the second part is some of the major transmission equipment, which completed the third part is the back-end is mainly composed of carrier network control of display devices, including back-end equipment can be further divided into central control equipment and control equipment. Front and back-end equipment form in several ways, linkages between them through the cable, fiber optic or microwave and other means to achieve.

Internet security systems combined with corporate status and characteristics of the industry, and traditional lack of communication equipment for today's safety requirements, presents a can monitor the video and audio records, environmental data collection, video commanding height, multifunctional alarm transport combine new intelligent platform. This system can achieve prevention, monitoring, emergency dispatching, scheduling needs for emergency treatment after a series of safety production. Internet monitoring system can realize the scene control, data acquisition, emergency monitoring and other business to uniform maintenance and manage integrated platform

On problems in the large-scale implementation of the Internet of things, operators with its network coverage, became the first choice of reliable partners. It is well known that operators ' networks all over China, for many years, in the community's public image has also been recognized by the masses. The sensor input, depending on the circumstances, connected to the operators ' networks, forms can use wireless or wired access.

Overall system design principle is to consider the technology to be able to have some forward-looking to ensure reliable security features and performance in order to ensure future upgrades to expansion, the system is extensible.

For the system with reduced coupling between modules, so when you design with hierarchical structure to be followed, by way of this design, and uses remote coding and transmission control technology, can perform hierarchical monitoring and management on site. Video surveillance, local storage, alarm linkage, such means as multiplex communication in parallel into the same under a unified management console, and each module can run on its own and can coordinate.

Equipment selection taking into account several aspects.

- Modular, considering future upgrades possible expansion of the system, whether it is hardware or software development, selected for both standard or generic device.

$\checkmark$ Select the device integration features, more convenient for operators and maintenance and repair.

- Because the landscape is diverse and complex, so the selected industrial unit shall be not subject to environmental impact, with high reliability. 
$\checkmark$ in the affordable range of devices with high-performance, advanced and require a reliable and practical.

Data network is primarily designed for the use of RFID and other real-time information collection, and into Internet gateways, Internet gateways will integrate information, and encapsulated into an agreed form, then send it to a wireless router. Wireless router is mainly used to access the network, and as a client to the tunnel server VPDN connection is established.

Data network components tend to be more clear, the main component of Internet gateway, sensor networks, wireless router. Its composition structure is shown in the following figure in these modules, sensor networks is one of the core part of its components generally have infrared sensors, temperature sensors, humidity sensors, video servers.

Future trend of network development is the spread of digital technology, far-reaching today's analog mode has been unable to meet the future market demand. Analog mode major mode of transmission was analog input/output, which originated from the baseband-disk video recorders work. But in the future, information and digital resource sharing model simulation communications development model is no longer able to satisfy. Digital network server's main application is implemented in the network monitoring system, this system can realize remote monitoring. Digital video service, resource sharing, multiplexing, software based on hardware is fully open source, implement systematic, high speed network video transmission. Of course, the video is just one of the basic functions, network video server can achieve deeper levels of information to promote the systematization of Web video services, and application development. Online video service, regardless of the content from the service, on application, on the future development model, has a broad prospect, is of far-reaching significance.

At the receiving node, or identity of the router server checks the user name and password pair to decide to allow or deny the connection request, and then sends to the requesting party to reject or accept the message.

PAP is not a strong authentication, when PAP is used by the link sends passwords in plaintext and therefore cannot prevent trial and error attacks and replay attacks, remote node controls the timing and frequency of login attempts. Router challenge party HQ will calculate the hash and compares the hash value sent RS, if these two values the same, HQ will send RS link response, as shown in Figure 1

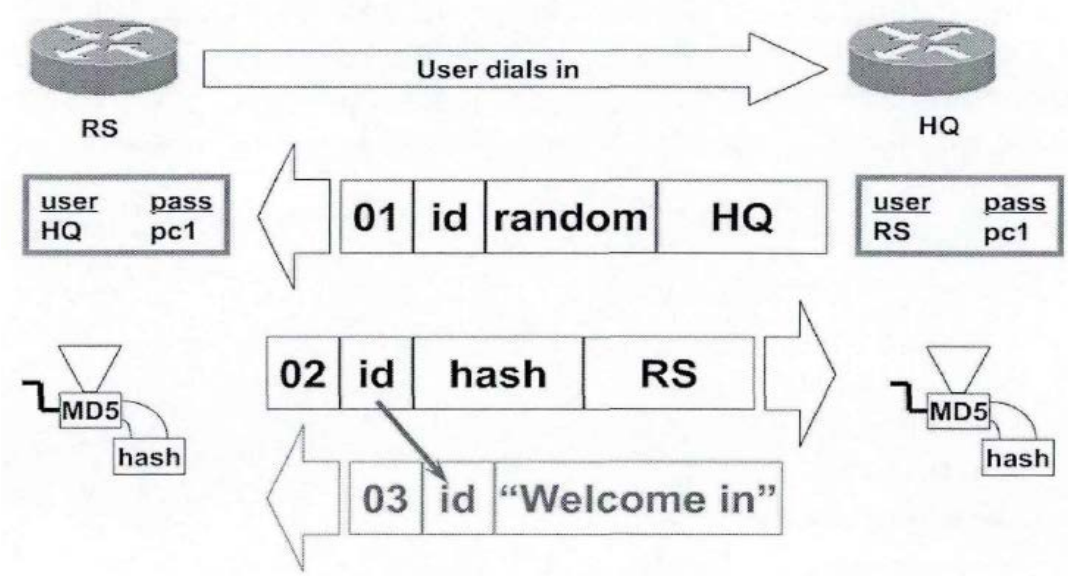

Fig.1. HQ building links

\section{Congestion control based on application layer}

Management and control system based on VPDN is a typical application of Internet of things, this application can be built using Internet standards. Dang tube control of on like in normal work State Xia Shi, on bandwidth of actual requirements very small, but still will produced large of network flow, caused bandwidth resources of great waste Dang appeared alarm State Shi, on bandwidth resources of requirements moments upgrade is high, this burst sex, and emergency sex of flow on network resources proposed has high of requirements, existing of QoS mechanism cannot 
meet real networking of this special needs. Presents a congestion control algorithm based on application layer, which can meet the requirements for control systems, ensure reasonable distribution of bandwidth of maximum probability and on-demand.

If a host sends a large amounts of data in port on host. Error occurred but the Middle needs to immediately suspend the execution of the program. Then the user can use the break command. If the emergency is not to identify urgent data, then the break command will be stored in the cache of the last, until all the data is processed after the break command in an application that was submitted to the on-side. So that you do not meet priority urgent data results.

In traditional data communications network in the, especially in Ethernet in the, prevent congestion main in three a level achieved, first a is data chain Road layer, main used heavy biography strategy, and mess sequence cache strategy, and confirmed strategy and flow control strategy second a is network layer, main used virtual circuit and data reported strategy, and group queued and service strategy, and group discarded strategy, and routing algorithm and group survival management third a is transmission layer, main used heavy biography strategy, and mess sequence cache strategy, and confirmed strategy, and flow control strategy and determine timeout strategy.

When monitoring equipment was in working condition, monitoring information is not uploaded to control processing center for processing and exchanging, only when the device is in the alarm State being monitored, and starts sending information and switching modules. The algorithm consists of three main modules and some auxiliary modules, three main functional modules of a selection of control center module, algorithm module launch, sending module.

\section{References}

[1] Cao Juying. Research and implementation based on the VPDN L2TP networks. information. 2009

[2] Yang Jianjun. Research of network authentication technology based on RADIUS protocol. Journal of Zhejiang University. 2005.2

[3] T. Joachims. Learning to Classify Text using Supprot Vector Machines: Methods, Theory, and Algorithms, Ph D. Dissertation, Department of Computer Science, University of Dortmund, 2000: 63 69

[4] J.A.K. Suykens, T.V. Gestel, J.D. Brabanter, et al. Least Squares Support Vector Machines. River Edge, NJ: World Scientific, 2002: 47 62

[5] C. Gold, P. Sollich. Model Selection for Support Vector Machine Classification Neuro computing, 2003, 55(1-2): 221 249

[6] S.S. Keerthi. Efficient Tuning of SVM yper parameters using Radius/Margin Bound and Iterative Algorithms. IEEE Trans. Neural Networks, 2002, 13: 1225 1229

[7] K. Ito, R. Nakano. Optimizing Support Vector Regression Hyperparameters Based on CrossValidation, Proc. Int. Joint Conf. Neural Networks, Portland, Oregon, 2003

[8] A. Smola. Hilbert Space Methods: Basics, Applications, Open Problems. RSISE Control Theory Seminar, 2002. http://sml.nicta.com.au/ smola/ 